\title{
Assessment of IL-10/IL-6 ratio in differential diagnosis of primary vitreoretinal lymphoma - a case series report
}

\section{Weronika Szliwa' ${ }^{1}$ Wojciech Adamski' ${ }^{1}$ Dariusz Wawrzyniak' ${ }^{2}$ Ewa Mizera-Nyczak³ ${ }^{3}$ Aldona Kaczmarek ${ }^{4}$, Katarzyna Rolle ${ }^{2}$, Andrzej Marszałek ${ }^{3}$, Iwona Rospond-Kubiak ${ }^{1}$}

'Department of Ophthalmology, Poznan University of Medical Sciences, Poland

2Department of Molecular Neurooncology, Institute of Bioorganic Chemistry, Polish Academy of Science in Poznan, Poland

${ }^{3}$ Department of Cancer Pathology and Prophylaxis, Greater Poland Cancer Centre, Poznan, Poland

${ }^{4}$ Flow Cytometry Laboratory, Greater Poland Cancer Centre, Poznan, Poland

\begin{abstract}
Aim of the study: To analyze the clinical applicability of interleukin 10 (IL-10) and interleukin 6 (IL-6) ratio assessment in intraocular fluids for the diagnosis of vitreoretinal lymphoma.

Material and methods: The data of the patients who underwent a diagnostic vitrectomy or anterior chamber fluid aspiration because of the clinical suspicion of vitreoretinal lymphoma at the Department of Ophthalmology, Poznan University of Medical Sciences (PUMS), between 2016 and 2019, were retrospectively reviewed. All patients underwent full ophthalmological examination including ultrasound prior to the surgery. Routine histopathology examination and flow cytometry were performed in obtained vitreous samples or aqueous humor aspirate as well as the evaluation of IL-6 and IL-10 ratio (IL-10 : IL-6) with the ELISA assay.
\end{abstract}

Results: There were 7 patients: 3 women, 4 men in the mean age of 67 years (range: 60-81 years). In 2 cases, primary vitreous involvement was suspected, and in another 5 , secondary. In 4 cases DLBCL (diffuse large B-cell lymphoma) was diagnosed. In 4 patients, the IL-10/IL-6 ratio was above 1.0, in 3 others - below 1.0. The median follow-up was 23 months (range: $2-82$ months). 5 lymphoma patients died 13 months after ophthalmic diagnosis due to disease progression.

Conclusions: The assessment of IL-10 and IL- 6 ratio may be a valuable tool facilitating the diagnosis of vitreoretinal lymphoma in some patients.

KEY WORDS: lymphoma, uveitis, interleukin 10, interleukin 6, vitreous biopsy.

\section{INTRODUCTION}

Lymphomas, primarily located within the eye and eye socket (mainly in the orbit or conjunctiva), are usually MALT lymphomas and carry a good prognosis. The most common intraocular lymphoma is primary vitreoretinal lymphoma (PVRL), which is a subtype of central nervous system lymphoma (CNSL) with usually a bad prognosis [1]. Vitreous involvement may precede the involvement of the CNS in some patients for a few months or years and PVRL often occurs concurrently with already diagnosed CNSL.

The etiology of non-Hodgkin's lymphomas still remains unclear but some environmental, immunological, and infectious factors were reported to have an influence on their development [2].

Ophthalmic signs and symptoms of intraocular lymphoma are non-specific and often pose a great challenge to a cli- nician. Patients normally would report blurred vision, metamorphopsias, reduced visual acuity, pain of the involved eye or headaches [3]. The typical sign of PVRL is the presence of cells in the anterior chamber or in the vitreous together with its opacity (Figure 1) [4]. Lymphomas can also imitate other intraocular tumors, retinal or choroidal dystrophies, as well as infectious or non-infectious uveitis. In primary vitreoretinal lymphoma (PVRL), there are infiltrates located at the retinal pigment epithelium layer and they are visible in OCT. They appear as multifocal, small, creamy subretinal lesions with a pigment on the surface at ophthalmoscopy examination. Additionally, neuroretinitis-like symptoms could be observed: optic nerve disc edema and vascular sheaths or vitreous cellular infiltrates.

To establish the final diagnosis a detailed ophthalmological examination is needed with some additional imaging 
tests, i.e. fundus autofluorescence, fluorescein or indocyanine green angiography, ultrasonography and OCT. Other procedures such as CNS imaging, bone marrow or lymph node biopsy are necessary to confirm systemic involvement, if suspected.

However, diagnostic biopsy of the vitreous, which can be performed by fine-needle aspiration biopsy (FNAB) of subretinal lesions or during pars plana vitrectomy (PPV), still remains the most reliable diagnostic tool in the diagnosis of intraocular lymphoma.

For patients with B-cell lymphoma, the most common markers are CD 19, CD 20, CD 22, $\kappa$ and $\lambda$ light chains. The neoplastic nature of $\mathrm{B}$ lymphocytes is evidenced by clonality, i.e. the restriction of the production of $\kappa$ and $\lambda$ immunoglobulin light chains, which means some type is predominantly produced.
Interleukin 10 is an inhibiting factor of pro-inflammatory cytokine synthesis. It was previously reported that its level increases as a result of stimulation of lymphoma cells. On the other hand, interleukin 6 is a pro-inflammatory cytokine that stimulates the production of acute phase proteins $[5,6]$ and prevails in various types of uveitis.

\section{AIM OF THE STUDY}

The aim of this study was to determine the clinical applicability of IL-10 to IL- 6 ratio assessment in the protocol for the differential diagnosis of vitreoretinal lymphoma.

\section{MATERIAL AND METHODS}

The medical records of the patients who entered the protocol for the diagnosis of vitreoretinal lymphoma at the Department of Ophthalmology, PUMS, between 2016 and 2019,
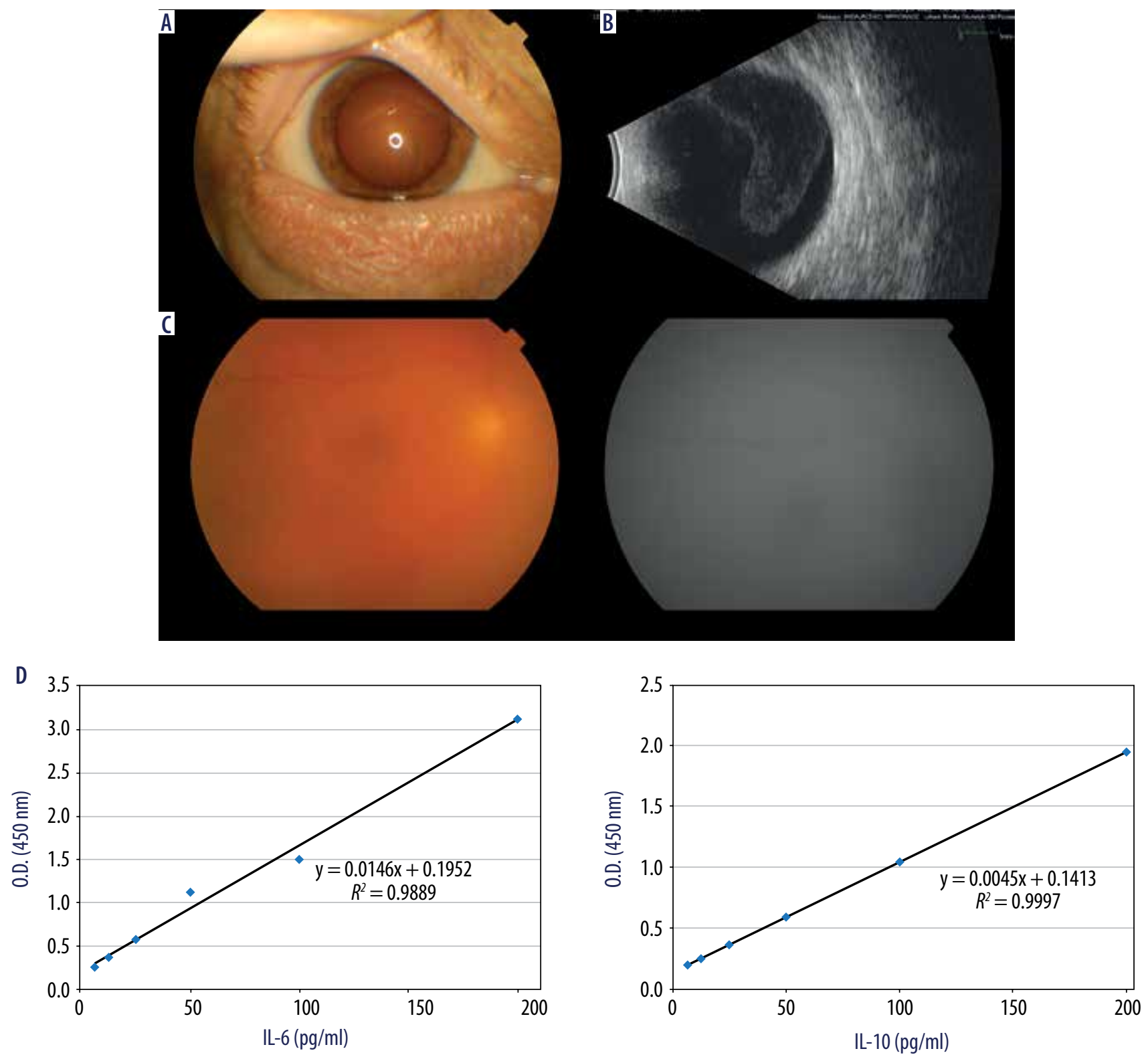

Figure 1. Photograph of the anterior segment of the eye (A), hyperechogenic, massive opacifications in the vitreous (B), blurred fundus view on ophthalmoscopy (C), interleukin 6 and 10 (D) profile which confirmed the diagnosis of DLBCL 
were retrospectively reviewed. After a complete ophthalmological examination, ultrasonography, OCT and CNS imaging, if applicable, all patients underwent diagnostic $25 \mathrm{G}$ pars plana vitrectomy (in 6 cases) or aqueous humor aspiration (in 1 case).

Flow cytometry of the undiluted sample (i.e., taken without infusion) was performed within 30 minutes from collection. The tested material was vitreous aspirate or anterior chamber aspirate in 1 patient. Biomaterial was analyzed in a BD FACSCanto TM Cytometer with the BD FACSDiva program.

In each sample IL-10 and IL- 6 cytokine concentrations were determined using the enzyme-linked ELISA, with the diagnostic kits Human IL-10 ELISA Kit (catalog number 950.060.192, DIACLONE SAS, France) and Human IL-6 ELISA Kit (catalog number 950.030.192, DIACLONE SAS, France), in ten replicates.

\section{RESULTS}

We identified 7 patients: 3 women, 4 men with the mean age of 67 years (range 60-81 years). In 5 patients the vitreous spread of diffuse large B-cell lymphoma (DLBCL) was diagnosed, in 2 other patients the lymphoproliferative disease was excluded.

In 1 patient the aqueous humor was collected for analysis because he was under systemic treatment for CNS lymphoma and he presented painful blind eye, high intraocular pressure and a mature cataract with no fundus view. Cyclocryotherapy was performed to lower the pressure and the eye globe was salvaged with anti-inflammatory treatment. Four months later, we observed opacification of the vitreous in the fellow eye, which was accompanied by a general relapse.

Summing up, in 4 cases, the interleukin 10 to 6 ratio was above 1.0, in 3 patients it was lower than 1.0. The median follow-up was 23 months (range: $2-82$ months). During this time, 5 patients died due to disease progression in the median time of 13 months.

Data of the patients and diagnoses are listed in Table I.

\section{DISCUSSION}

Histopathology and flow cytometry of the vitreous sample do not always confirm or exclude the clinical suspicion of primary vitreous lymphoma (PVRL). According to the literature, the percentage of false negative results is about $30-45 \%$ [6]. It means that the diagnosis could be certain only in about $50-60 \%$. The vitreous is a paucicellular material, so proper processing of the sample seems to be essential for the accuracy of a diagnosis based on the biopsy. If the time of transport and preparation of the sample exceeds 1 hour, a preservative, e.g. PreservCyt, HOPE, should be used in order to perform cytological and molecular analysis as well as flow cytometry at the same time $[7,8]$. Prior to a biopsy, general/topical steroid therapy should be discontinued due to the cytolytic activity for lymphocytes, which may affect the final results [6].

According to the majority of published analyses on the subject, the ratio of IL-10 : IL-6 is > 1.0 in intraocular fluids in patients with primary intraocular lymphoma, although there are some reports that it might be reduced below 1.0. [5, 9-11]. Costopoulos et al., in a retrospective, multicenter-case series study, analyzed 510 samples using a Cytometric Bead Array kit (CBA, BD Biosciences). In this study, the ratio of IL-10 : IL-6 was $<1.0$ and did not exclude intraocular lymphoma in each patient, so the authors proposed a comparison of the result with the clinical probability of lymphoma (the Interleukin Score for Intra-Ocular Lymphoma Diagnosis, ISOLD), and they obtained a method with high sensitivity (Se) and specificity (Sp) $[9,10]$. Fisson et al. examined samples from 87 patients, with primary intraocular lymphoma (PIOL), CNS lymphoma, uveitis and from a control group with non-inflammatory disease. They determined interleukins IL-2, IL-4, IL-6, IL-10, interferon c (IFN-c) and tumor necrosis factor $\alpha$ (TNF- $\alpha)$. IL-2, IL-4 and TNF- $\alpha$ were not detected in any sample. The combination of IL-10/IL-6 and IL-10/IFN-c ratios was helpful in distinguishing PIOL or oculocerebral lymphoma (OCL) from uveitis. They also compiled the CBA and ELISA methods, and IL-10 concentrations were highly correlated [11]. Cochat-Potilloux et al. found that a cutoff of $65 \mathrm{pg} / \mathrm{ml}$ for IL-10 in the vitreous samples was associated with sensitivity (Se) of $93 \%$ and specificity (Sp) of $100 \%$. In the aqueous humor samples, a cutoff of $30 \mathrm{pg} / \mathrm{ml}$ gave Se of $78 \%$ and Sp of $97 \%$. Additionally, a combination of results with the IL-10 : IL-6 ratio greater than 0.6 increased the Sp to $100 \%$ [12].

Of note, the results may be affected by the amount of the tested sample and other limitations (retrospective study). IL-10 concentration in the aqueous humor and the vitreous may vary also in the same eye. In our study, interleukin level determination was performed always parallel to flow cytometry and histopathology examination. In most of the analyzed cases, the IL-10 : IL-6 ratio confirmed the clinical suspicion of a secondary infiltration of lymphoma in the eye despite general remission of the disease and negative histopathology. In 3 cases, despite the inconclusive result of histopathology or flow cytometry (B-cell domination but without $\lambda$ or $\kappa$ chain expressions in 2 cases and in 1 case domination of activated T lymphocytes with co-occurrence of B-cells with domination of $\kappa$ chains), the IL-10 : IL- 6 ratio $>1.0$ resulted in the diagnosis of secondary DLBCL, while in the other 2 cases IL-10 : IL- 6 ratio $<1.0$ was crucial to the exclusion of lymphoproliferative disease after taking into consideration the whole patient's medical history. In 1 case, the IL-10 : IL-6 ratio was $>1.0$ but a choroidal tumor of unknown etiology was diagnosed and in 1 case the IL-10 : IL- 6 ratio was $<1.0$ (anterior chamber fluid sample) and there were no cells for immunophenotype evaluation in flow cytometry despite the strong clinical features of IOL.

As the diagnosis of vitreoretinal lymphoma is always associated with a serious prognosis for the patient and the risk of CNS involvement, the diagnosis of the primary location in the eye is related to the need for systemic or local chemotherapy. In metastatic lymphomas, systemic chemotherapy is usually required, and if the disease activity is limited to the eye only, external-beam radiotherapy (EBRT) is recommended [13]. For 


\begin{tabular}{|c|c|c|c|c|c|c|c|}
\hline $\begin{array}{l}\frac{\overrightarrow{3}}{3} \\
\text { 흥 }\end{array}$ & $\begin{array}{l}\text { 喜 } \\
\text { 章 }\end{array}$ & 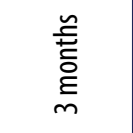 & 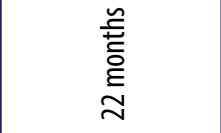 & 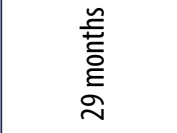 & 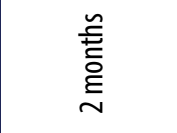 & $\begin{array}{l}\text { 美 } \\
\text { 音 } \\
\text { g }\end{array}$ & 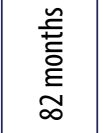 \\
\hline 网 & 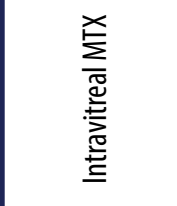 & 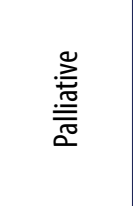 & 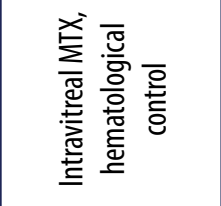 & 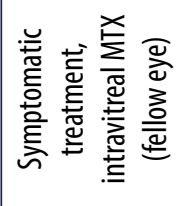 & 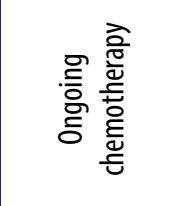 & 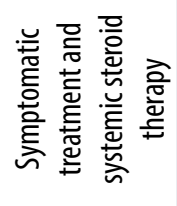 & 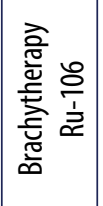 \\
\hline$\stackrel{\stackrel{\bullet}{\Perp}}{\text { \& }}$ & $\stackrel{\circ}{\stackrel{\leftrightarrow}{\wedge}}$ & $\stackrel{\circ}{\stackrel{\circ}{\wedge}}$ & $\stackrel{ }{\stackrel{ }{\Lambda}}$ & $\stackrel{\circ}{\vec{v}}$ & $\underset{\wedge}{\stackrel{ }{x}}$ & $\stackrel{\circ}{\vec{v}}$ & $\stackrel{\circ}{\mathrm{v}}$ \\
\hline 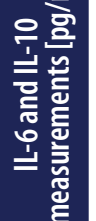 & 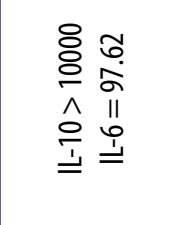 & 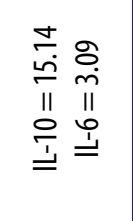 & 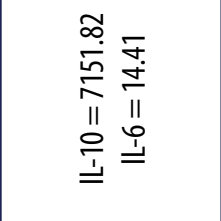 & 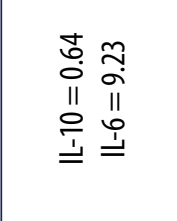 & 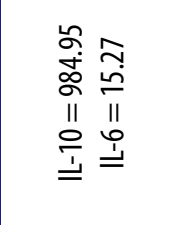 & 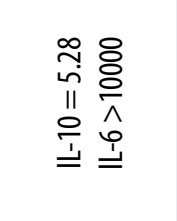 & 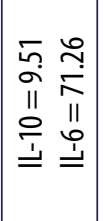 \\
\hline 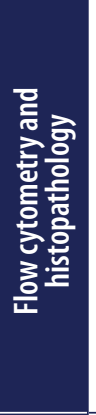 & 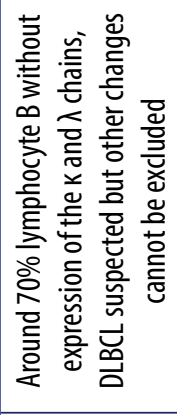 & 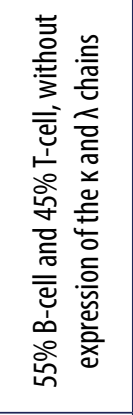 & 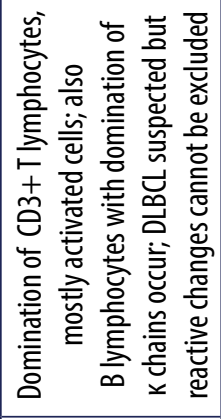 & 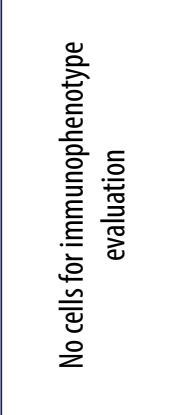 & 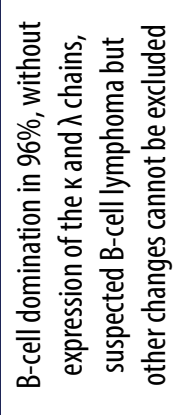 & 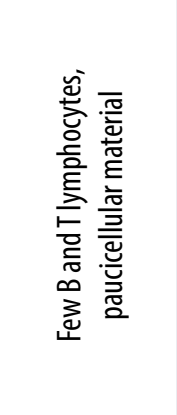 & 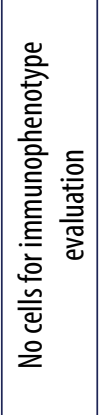 \\
\hline 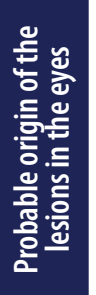 & 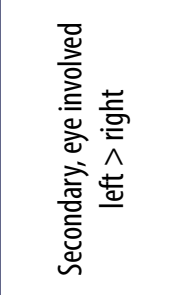 & 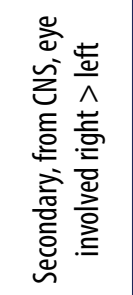 & 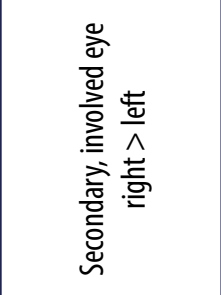 & 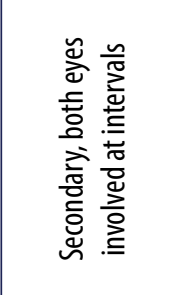 & 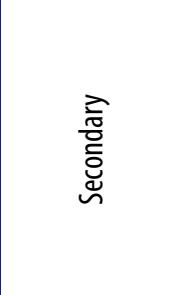 & $\begin{array}{l}\text { 촣 } \\
\text { 产 }\end{array}$ & 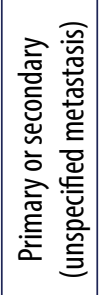 \\
\hline 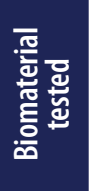 & 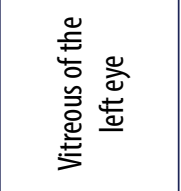 & 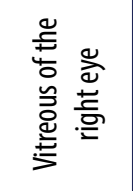 & 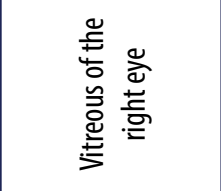 & 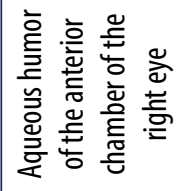 & 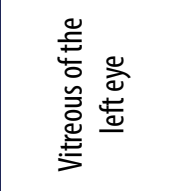 & 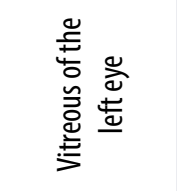 & 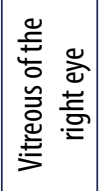 \\
\hline 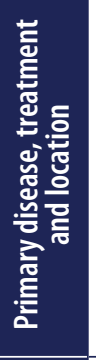 & 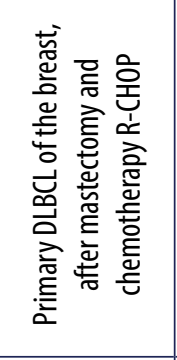 & 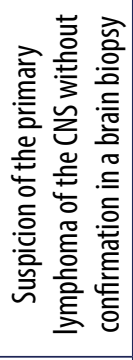 & 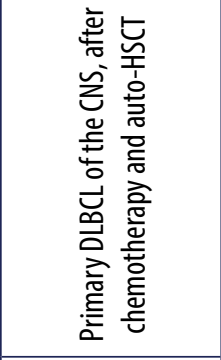 & 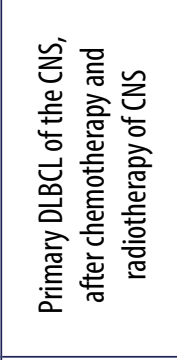 & 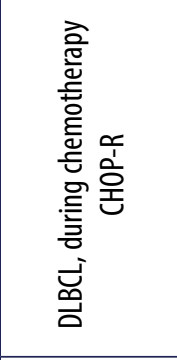 & 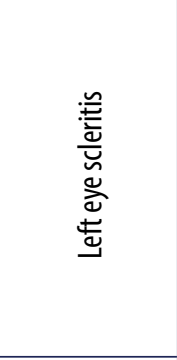 & 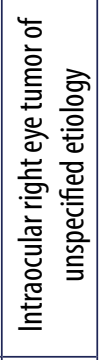 \\
\hline 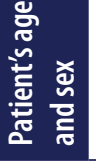 & 온 & is & $\stackrel{8}{\Sigma}$ & $\frac{8}{i}$ & $\sum_{i}^{\mathbb{J}}$ & $\begin{array}{l}\infty \\
\stackrel{4}{4}\end{array}$ & ${ }_{i}^{8}$ \\
\hline
\end{tabular}


our four patients diagnosed with IOL or with strong suspicion of IOL we used intraocular methotrexate injections. General treatment with an appropriate chemotherapy protocol was performed at a hematological ward.

\section{CONCLUSIONS}

The assessment of IL-10 : IL-6 ratio seems to be a valuable test in differentiating between inflammatory lesions and primary intraocular lymphoma. It could be used as support- ive tool to make the final diagnosis faster. However, due to the lack of clear guidelines and interleukins ratio cut-off level, the interpretation of the results and final diagnosis need to be supported by the whole clinical history of the patient and results of additional tests and examinations.

\section{DISCLOSURE}

The authors declare no conflict of interest.

\section{References}

1. Amin MB, Edge SB, Greene FL, et al. AJCC Cancer Staging Manual, Springer International Publishing, 2018: 931-937.

2. Gajewski P. Interna Szczeklika, Medicina interna, basada en la evidencia. Medycyna Praktyczna 2015: 966-970.

3. Araujo I, Coupland SE. Primary vitreoretinal lymphoma, a review. Asia Pac J Ophthalmol (Phila) 2017; 6: 283-289.

4. Cho BJ, Kim DY, Park UC, et al. Clinical features and treatment outcomes of vitreoretinal lymphoma according to its association with CNS lymphoma. Ocul Immunol Inflamm 2018; 26:365-371.

5. Cassoux N, Giron A, Bodaghi B, et al. IL-10 measurements aqueous humor for screening patients with suspicion of primary intraocular lymphoma. Invest Ophthalmol Vis Sci 2007; 48: 3253-3259.

6. Barry RB, Tasiopolou A, Murray PI, et al. Characteristic optical coherence tomography findings in patients with primary vitreoretinal lymphoma: a novel aid to early diagnosis. Br J Ophthalmol 2018; 102: 1362-1366.

7. Sagoo MS, Mehta H, Swampilla AJ, et al. Primary intraocular lymphoma. Surv Ophthalmol 2014; 59:503-516.

8. Jahnke K, Thiel E, Abrey LE, et al. Diagnosis and management of primary intraocular lymphoma: an update. Clin Ophthalmol 2007; 1:247-258.

9. Kuo DE, Wei MM, Knickelbein JE, et al. Logistic regression classification of primary vitreoretinal lymphoma versus uveitis by interleukin 6 and interleukin 10 levels. Ophthalmology 2020; 127: 956-962.

10. Costopoulos M, Touitou V, Golmard JL, et al. ISOLD: A New Highly Sensitive Interleukin Score for Intraocular Lymphoma Diagnosis. Ophthalmology 2016; 123: 1626-1628.

11. Fisson S, Ouakrim H, Touitou V, et al. Cytokine profile in human eyes: contribution of a new cytokine combination for differential diagnosis between intraocular lymphoma or uveitis. PLoS One 2013; 8: e52385.

12. Pochat-Cotilloux C, Bienvenu J, Nguyen AM, et al. Use of a threshold of interleukin-10 and IL-10/IL-6 ratio in ocular samples for the screening of vitreoretinal lymphoma. Retina 2018; 38: 773-781.

13. Romanowska-Dixon B, Jager MJ, Coupland SE. Onkologia okulistyczna. Wyd. Lek. PZWL, Warszawa 2019; 459-469.

14. Fukunaga H, Kaburaki T, Shirahama S, et al. Analysis of inflammatory mediators in the vitreous humor of eyes with pan-uveitis according to aetiological classification. Sci Rep 2020; 10: 2783.

15. Kimura K, Usui Y, Goto H. Japanese Intraocular Lymphoma Study Group. Clinical features and diagnostic significance of the intraocular fluid of 217 patients with intraocular lymphoma. Jpn J Ophthalmol 2012; 56: 383-389.

16. Ohta K, Sano K, Imai H, et al. Cytokine and molecular analyses of intraocular lymphoma. Ocul Immunol Inflamm 2009; 17: 142-147. 